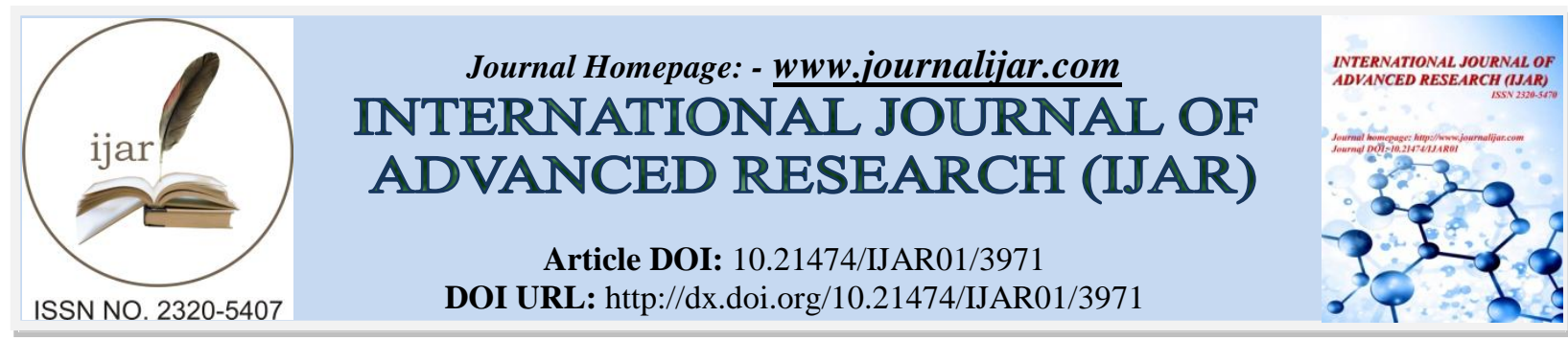

RESEARCH ARTICLE

\title{
MORPHOLOGICAL VARIATIONS OF THE CONDYLE USING CONE BEAM COMPUTED TOMOGRAPHY - A RETROSPECTIVE STUDY.
}

\author{
Dr. Saraswathi Gopal. K, Dr. Vani Anusha. A and Dr. Mahesh Kumar. P.
}

Prof And Head Of The Department Of Oral Medicine And Radiology .Meenakshi Ammal Dental College And Hospital, Chennai, India.

\section{Manuscript Info}

Manuscript History

Received: 13 February 2017

Final Accepted: 08 March 2017

Published: April 2017

Key words:-

Tempomandibular joint; condylar morphology; Cone Beam Computed Tomography.

\begin{abstract}
Background: Cone beam computed tomography (CBCT) can detect morphological variations of the condyle accurately in three dimensions. This study was conducted to assess the morphology of the condyle using three dimensional cone beam computed tomography in coronal and sagittal sections.

Materials and method: This was a retrospective study where cone beam computed tomography images from radiology archives were analysed to assess the morphology of right and left condyles with planmeca promax 3D using romexis software. 50 images (100 condyles bilaterally) of the patients between age group 15 to 45 yrs (Mean age $30 \mathrm{yrs}$ ) formed our study group. Qualitative data analysis was done using chi square test.

Results: In coronal view 4 types of condyles were seen type I convex 49\%; type II flat 21\%; type III round 23\% and typeIV angled $7 \%$ ( $p$ value $<0.05$ ). In sagittal view three types of condyles were elucidated type I round $78 \%$, type II flat $8 \%$ and type III beaked $14 \%$ ( $p$ value $<0.05$ ).

Conclusion: Morphological variations of condyle in both sagittal and coronal sections using cone beam computed tomography have been observed along with varied distribution and prevalence among different age groups. Radiographic evaluation of condylar morphology aids in an early detection of the temporomandibular joint disorders and functional alterations which is facilitated more accurately in three planes by cone beam computed tomography.
\end{abstract}

Copy Right, IJAR, 2017,. All rights reserved.

\section{Introduction:-}

Anatomical knowledge of the temporomandibular joint is one of the foundations for the clinical practice that allows the understanding of pathological alterations, diagnostic evaluation and appropriate choice of therapeutic paths ${ }^{1}$.The temporomandibular joint is a unique joint that can be affected by different kinds of hard and soft tissue abnormalities $^{2}$ The bony components of TMJ need a careful study since the changes in these components may imply functional disorders and pain ${ }^{1}$. Temporomandibular joint disorders are a subdivision of musculoskeletal pathologies which act as a source of orofacial pain of non dental origin ${ }^{3}$. These temporomandibular joint disorders are frequently associated with certain degenerative bony changes that include; Flattening, Erosion, Osteophytes etc, all of which involve the bony components of TMJ.

Corresponding Author:- Dr. Saraswathi Gopal. K, Address:- Prof And Head Of The Department Of Oral Medicine And Radiology .Meenakshi Ammal 1485 
Within the bony components, there is a large topographical variability between the condyle and mandibular fossa which is affected by the shape of the condyle that suffers or is subjected to changes due to bone remodelling ${ }^{1}$.This is because the condyle being the primary growth centre in the mandible, responds to continuous stimuli throughout the remodelling process and hence plays a role in the final dimensions of the adult mandible ${ }^{4}$. Radiographic examination of TMJ is the part of clinical assessment and is important for evaluating the abnormalities and bony changes that affect the TMJ. CBCT, due to its high spatial resolution and low radiation dose when compared to CT is considered the modality of choice for imaging the osseous components of TMJ. The present study aims at evaluating the morphological variations in the condyle using CBCT and to assess the prevalence of different morphologies among various age groups.

\section{Materials and Method:-}

A retrospective observational study was conducted for a duration of two months (August- October, 2016) in the Department Of Oral Medicine And Radiology of a private dental college in Chennai. Sample size was calculated to be 50 images keeping the power of the study $90 \%$ and alpha error at 5\%. Fifty CBCT images of the condyle (100 images bilaterally), were retrieved from the radiology archives. These images were previously obtained for the radiographic evaluation of impacted third molars and bone assessment for the implant placement. Images of the patients between 15 and 45 yrs were collected. The full skull CBCT images of these 50 patients (100 condyles) were evaluated by a single examiner. Intra examiner calibration was assessed performing test-retest exercises in 10 images of patients with intra examiner agreement of $95 \%$.

The images with radiographic artifacts, full skull images that had no condylar morphological details and patients with temporomandibular joint disorders were excluded.

Morphological variations in the condyle were assessed and morphology was classified further in coronal and sagittal views with planmeca promax 3D unit using Romexis software.

In the coronal section; the morphology of the condyle was categorized based on the classification given by Yale et $\mathbf{a l}(1966)^{5}$ as convex, round, flat and angled(Fig-1).

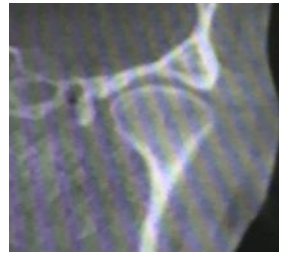

Convex

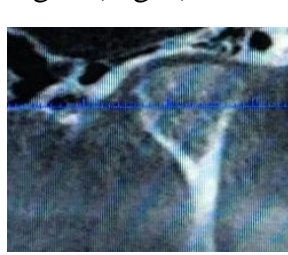

Round

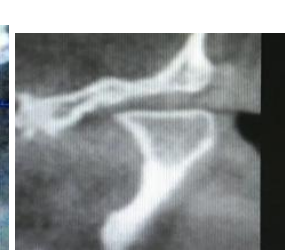

Flat

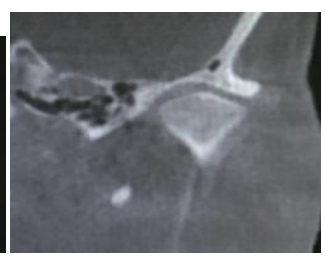

Angled

Fig 1:- Showing the four morphological variations of condyle in coronal view.

In the sagital view; the morphology was classified based on the classification given by Koyama et al (2007) ${ }^{6}$ as round, beak shaped and flat (Fig-2).

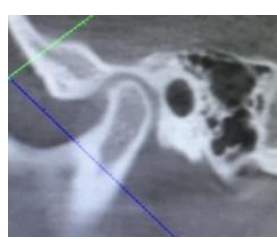

Round

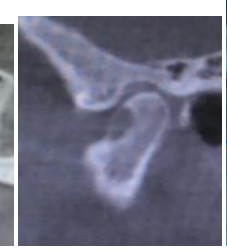

Beak shaped

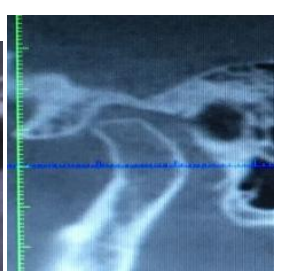

Flat

Fig 2:- Showing the morphological variations of condyle in the sagital view.

The sample was divided into three different groups, group I(15-24 yrs), group II(25-34 yrs), group III(35-45 yrs) based on the age. The prevalence of different condylar morphological patterns among these groups was studied.

Statistical analysis was carried out with SPSS version 16 software (IBM.corp.Chicago.,USA). Qualitative data analysis was carried out using chi square test. The results with $\mathrm{p}$ value $<0.05$ with $95 \%$ confidence level were considered to be significant in this study. 


\section{Results:-}

In coronal view, the most common morphology observed was convex $49 \%$ followed by round $23 \%$, flat $21 \%$ and angled type 7\% (Graph-1). In sagittal view, the most common pattern was Round $78 \%$ of total condyles followed by beak shaped $14 \%$ and flat $8 \%$ morphology (Graph-2). Erosions were not seen in our study in the sagittal view.

In the coronal view, among group I, nearly $38 \%$ of condyles examined had convex morphology, $30 \%$ had round morphology, $23.3 \%$ had flat condyles and only $8.33 \%$ were angled. In group II, $64.28 \%$ of condyles were of convex morphology, $10.71 \%$ had round morphology, $21.4 \%$ were flat condyles and only $3.57 \%$ showed beak shaped deformity. In group III, $66.67 \%$ of condyles were convex, $16.67 \%$ of condyles had round morphology and $8.33 \%$ of condyles showed flattening and equal percentage of them were angled (Graph-3)

The prevalence of convex morphology had increased from group I to group III significantly with a $\mathrm{p}$ value $<0.05$. There was no significant difference in the distribution of other morphologies among the three groups ( $\mathrm{p}$ value> $0.05)$.

In the sagittal view, majority of the subjects among group I had round morphology $85 \%$, whereas it is reduced to $75 \%$ and $50 \%$ among group II and group III subjects respectively (Graph-4). On the other hand, beaked morphology increased from $8.33 \%$ to $41 \%$ among group I to group III subjects ( $\mathrm{p}$ value< 0.05 ).

The following graph represents the most common condylar morphology in coronal view

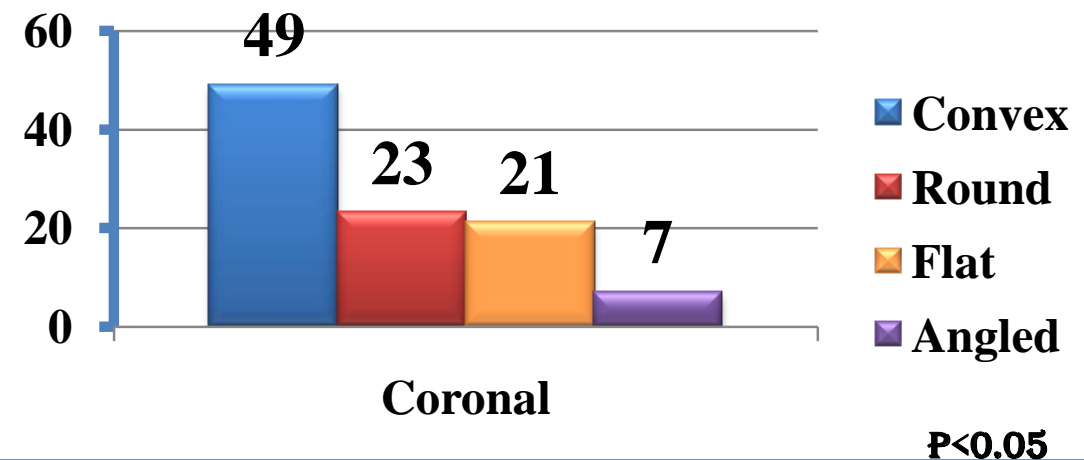

Graph 1:- representing different morphological variations in coronal view.

The following graph represents the most common condylar morphology in sagittal view

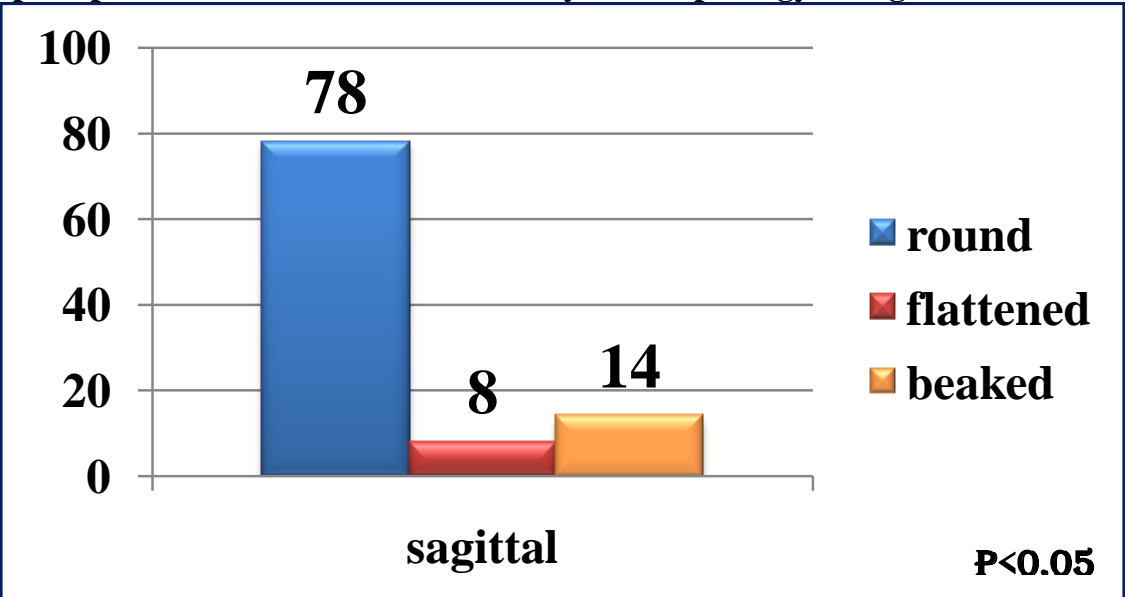

Graph 2:- representing different morphological variations in sagittal 
Morphology of condyle in three different age groups among the selected samples in coronal view

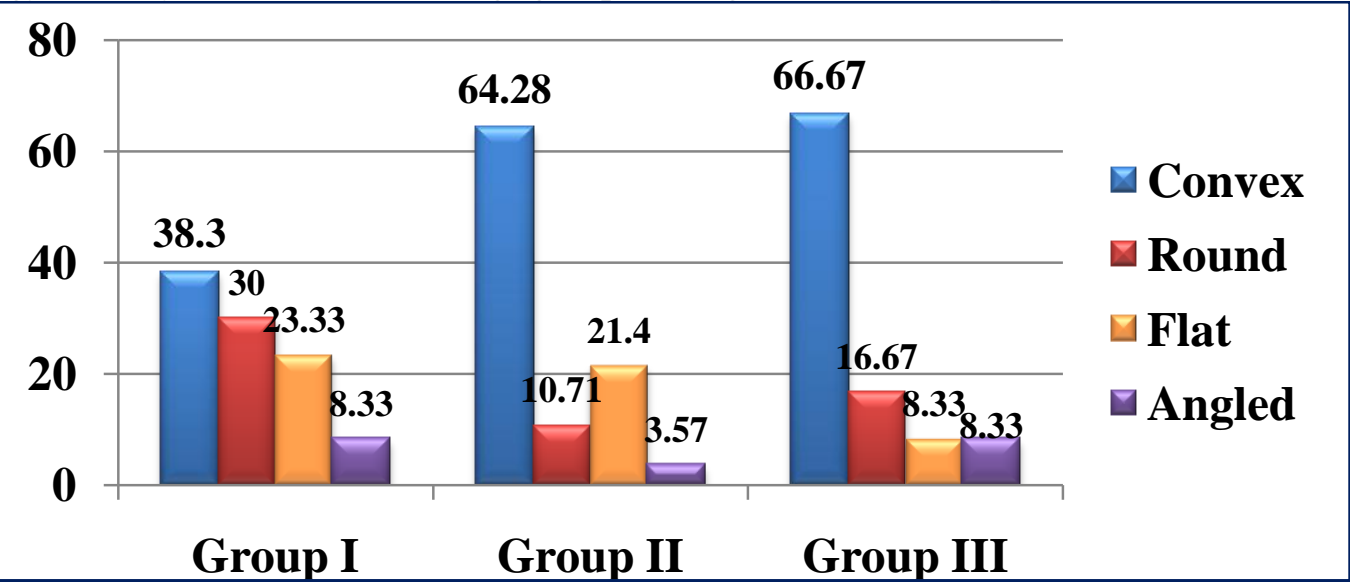

Graph 3:-. representing the prevalence of different morphologies in three different age groups in coronal view.

Morphology of condyle in three different age groups among the selected samples in sagittal view.

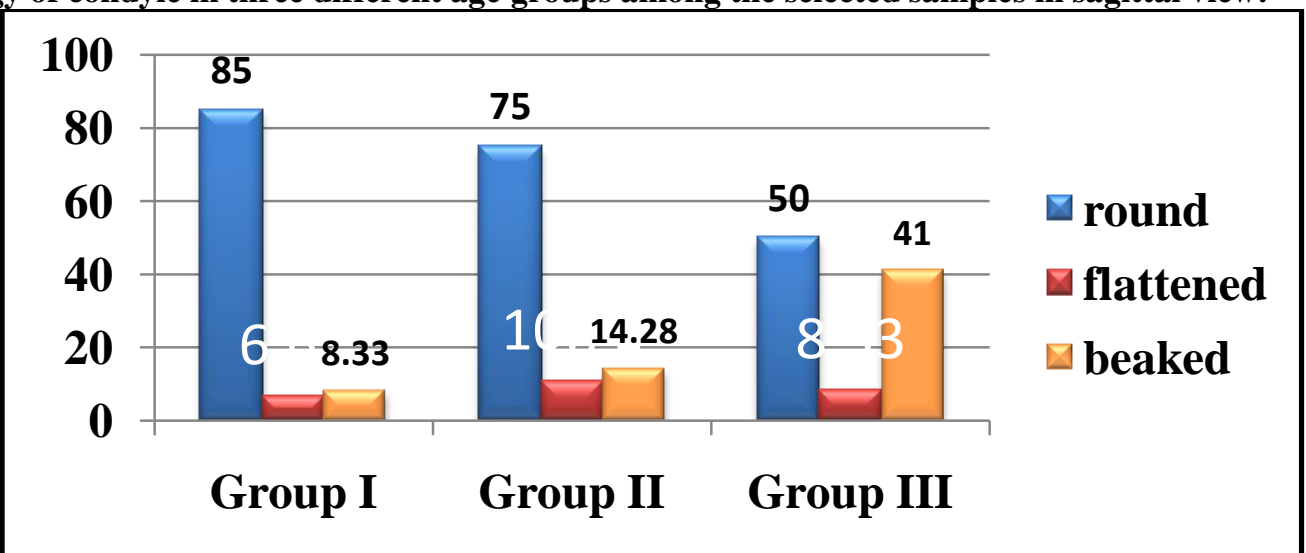

Graph 4:- representing the prevalence of different morphologies in three different age groups in sagittal view.

\section{Discussion:-}

Very few studies were conducted world-wide to assess the condylar morphology using three dimensional imaging. Furthermore, none as of date has been conducted to assess morphological variations along with their distribution and prevalence among different age groups using CBCT.

The osseous components of the TMJ are to be examined radiographically to assess their morphology, integrity and structural alterations. Degenerative bone changes that affect the TMJ can be evaluated by various radiographic methods for which a clear and precise image of TMJ is required and is difficult to attain from two dimensional imaging ${ }^{7}$.

Advances in CT has provided an increased accuracy in the diagnosis of degenerative bone changes by limiting the problems encountered during imaging that include superimposition of adjacent structures, different angulations of the condyle, presence of artifacts and mandibular movements during the examination ${ }^{8}$.It was suggested that CT could be reserved for ankylosis of TMJ, complex facial fractures, suspected tumors etc. considering the high dose of radiation and cost involved ${ }^{9}$.Thus, CBCT provides images with high diagnostic quality at a lower exposure and is cost effective when compared to CT.

The type of morphology of the condyle on imaging creates an initial environment, which is continuously modified throughout life based on the type of mastication of the individual and also due to the fact that this acts as a primary growth centre ${ }^{10}$. 
Diagnosis of the degenerative changes affecting the TMJ can be done more accurately using CBCT which provides images at a lower exposure than $\mathrm{CT}^{2} \mathrm{The}$ results of our study revealed that in coronal section, convex morphology was more commonly observed(49\%) followed by round(23\%). This was in accordance with the study conducted by Yale et.al(1966) ${ }^{5}$. However, in a study conducted by Subhashini et.al. the most common morphology observed was angled $(37.5 \%)$ in coronal view ${ }^{11}$.

In the sagittal view the most common morphology was round (78\%). This was in accordance with the study conducted by Subhashini et. al(2016) ${ }^{11}(71.9 \%)$. Unlike this study, erosions were not observed in our study in the sagittal view in any of the age groups among the selected samples.

In the present study, the significant increase in the prevalence of beak shape of condyle from group I to group III shows the association between the morphological pattern and the functional adaptation ${ }^{12}$. This association of condylar morphology and bony changes with physiological adaptation irrespective of age and gender was also in accordance with the study conducted by Naveen Kumar et al(2016) ${ }^{12}$. Morphology of the condyle contributes to the masticatory system. for instance, the beak shaped and angled condyles are usually associated with a hard bite ${ }^{9}$. Flattening may also result from overload on the TMJ and may be associated with involvement of masseter and temporalis muscles ${ }^{13}$.

However, our study has a limitation of unequal sample size distribution in different groups. Hence, further studies with extended wide range of sample and its equal distribution among the age groups and gender are suggested to correlate the morphological variations with degenerative changes or functional discrepancies.

\section{Conclusion:-}

Variation in the shape of the condyle may cause difficulty with radiographic interpretation. CBCT images provide the clinician with the opportunity to visualize the local and regional effects associated with morphological variations. Altered morphology of condyle may imply dysfunction, discrepancies, deformities and degenerative changes in TMJ which can be attributed not only to the senescence but also to other physiological alterations and adaptations. Thus, a thorough examination of the morphology of condyle and joint as a whole is essential which is radiologically guided more accurately by $\mathrm{CBCT}$. Early detection of morphological or functional alterations and abnormalities can be achieved using CBCT though the patients are clinically asymptomatic.

\section{References:-}

1. Ribeiro,E.C; Sanches, M.L; Alonso, L.G And Smith, R.L. Shape And Symmetry Of Human Condyle And Mandibular Fossa. Int.J. Odontostomat.,9(1):65-72,2015.

2. M Alkhader, Akuribayashi, N Ohbayashi, S Nakamura And T Kurabayashi. Usefulness Of Cone Beam Computed Tomography In Temporomandibular Joints With Soft Tissue Pathology. Dentomaxillofacial Radiology (2010) 39, 343-348.

3. ML Dos Anjos Pontual, JSL FREIE, JMN Barbosa, MAG Frazao, MM Fonseca Da Silveira. Evaluation Of Bone Changes In The Temporomandibular Joint Using Cone Beam CT. Dentomaxillofacial Radiology (2012) 41, 24-29.

4. May Al-Koshab, Prabhakaran Nambiar, Jacob John(2015) Assessment Of Condyle And Glenoid Fossa $\begin{array}{lllll}\text { Morphology Using } & \text { CBCT } & \text { In } & \text { South }\end{array}$ ONE10(3):E0121682.Doi:10.1371/Journal.Pone.0212682.

5. Yale SH, Allison BD, Hauptfuehrer JD. An epidemiological assessment of mandibular condyle morphology. Oral Surg Oral Med Oral Pathol 1966; 21: 169-177.

6. Koyama J, Nishiyama H, Hayashi T. Followup study of condylar bony changes using helical computed tomography in patients with temporomandibular disorder. Dentomaxillofac Radiol 2007; 36: 472-477.

7. K Tsiklakis, K Syriopoulos And HC Stamatakis. Radiographic Examination Of The Temporomandibular Joint Using Cone Beam Computed Tomography. Dentomaxillofacial Radiology (2014) 33, 196-201.

8. Zahra Dalili, Nasim Khaki, Seyed Javad Kia, Fatemah Salamat. Assessing Joint Space And Condylar Position In The People With Normal Function Of Temporomandibular Joint With Cone Beam Computed Tomography. Dental Research Journal Sep 2012; Vol 9; Issue 5.

9. K Honda, TA Larheim, K Maruhashi, Kmatsumoto And K Iwai. Osseous Abnormalities Of The Mandibular Condyle: Diagnostic Reliability Of Cone Beam Computed Tomography Compared With Helical Computed Tomography Based On An Autopsy Material. Dentomaxillofacial Radiology (2006) 35, 152-157. 
10. T A Larheim, A-K Abrahamsson, M Kristensen And L Z Arvidsson. Temporomandibular Joint Diagnostics Using CBCT. Dentomaxillofacial Radiology (2015)44,20140235.

11. Shubhasini AR, Praveen Birur N, Shubha G, Keerthi G, Sumsum P Sunny, Darshana S Nayak (2016).Condylar morphology on CBCT. MJDS, 1(1), 7-12.

12. Naveen Kumar.B, Saraswathi Gopal.K, Harsha Vardhan.B.G, Evaluation of bone changes and range of motion of the temporamandibular joint using cone beam computed tomography- a prospective cross sectional study, International Journal of Current Research,8(04).29737-29743.

13. K Ejima, D Schulze, A Stippig, K Matsumoto, D Rottke And K Honda. Relationship Between The Thickness Of The Roof Of Glenoid Fossa, Condyle Morphology And Remaining Teeth In Asymptomatic European Patients Based On Cone Beam CT Data Sets. Dentomaxillofacial Radiology (2013) 42, 90929410. 